\title{
Evidencias de validez factorial de la Escala de desesperanza de Beck en español con muestras clínicas y no clínicas
}

\author{
Alicia Edith Hermosillo-De la Torre; Cecilia Méndez-Sánchez; Fabiola González-Betanzos
}

How to cite this article:

Hermosillo-De la Torre, A.E., Méndez-Sánchez, C. \& González-Betanzos, F. (2020). Evidence for the Factorial Validity of the Beck Hopelessness Scale in Spanish with Clinical and non-Clinical Samples. Acta Colombiana de Psicología, 23(2), 148-158. http://www.doi.org/10.14718/ACP.2020.23.2.7

Recibido, octubre 10/2018; Concepto de evaluación, enero 18/2019; Aceptado, febrero 22/2019

\author{
Alicia Edith Hermosillo-De la Torre \\ Universidad Autónoma de Aguascalientes, Aguascalientes, México \\ ORCID: https://orcid.org/0000-0002-56362065 \\ Cecilia Méndez-Sánchez \\ Universidad Autónoma de Aguascalientes, Aguascalientes, México \\ ORCID: https://orcid.org/0000-0002-3079-5129 \\ Fabiola González-Betanzos" \\ Universidad Michoacana de San Nicolás de Hidalgo, Morelia, México \\ ORCID: https://orcid.org/0000-0003-4585-7211
}

\begin{abstract}
Resumen
El propósito del presente estudio fue evaluar la estructura interna de la adaptación al español de Escala de Desesperanza de Beck et al. (1974), dada su utilidad y relevancia en la predicción de conductas suicidas. Para esto, se analizaron las respuestas a la escala de 1260 estudiantes universitarios $(M=4.79 ; D T=4.29)$ y de una muestra clínica en la que participaron 150 jóvenes con intento de suicidio de alta letalidad $(M=8.51 ; D T=2.38)$. Se examinó la estructura interna por medio del Análisis factorial confirmatorio (AFC) en tres fases: en la primera, se comparó el modelo original con cuatro modelos encontrados en las diferentes adaptaciones al español; en la segunda, se tomaron en cuenta modelos que analizan la aquiescencia; y en la tercera, se hizo una validación cruzada de esos modelos con población clínica. Los resultados señalan que la escala es unidimensional tanto en el caso de las muestras clínicas $\left(\chi^{2}=154.84, g l=135, p<0.001, \mathrm{CFI}=0.99\right.$, TLI $=0.99$, RMSEA $=0.03$ ) como en la población universitaria; sin embargo, a esta última se le añadió un factor de método para el tratamiento de la aquiescencia $\left(\chi^{2}=252.14, g l=134, p<0.001\right.$, CFI $=0.95$, TLI $=0.94$, RMSEA $\left.=0.03\right)$. Los resultados muestran la importancia de utilizar análisis y modelos que consideren la naturaleza de los datos y las características de la muestra para aportar evidencias más sólidas para la validez de constructo.

Palabras clave: adaptación, desesperanza, validez, riesgo de suicidio, aquiescencia.
\end{abstract}

\section{Evidence for the Factorial Validity of the Beck Hopelessness Scale in Spanish with Clinical and non-Clinical Samples}

\begin{abstract}
The purpose of the present study is to evaluate the internal structure of the Spanish adaptation of the Beck Hopelessness Scale (Beck et al., 1974) given its usefulness and relevance in the prediction of suicidal behaviors. The responses to the scale of 1260 university students $(M=4.79, S D=4.29)$ and of a clinical sample in which 150 young people with suicide attempt of high lethality $(M=8.51, S D=2.38)$ participated were analyzed. The internal structure of the scale is examined by confirmatory factor analysis (CFA) in three phases. In the first phase, the original model is compared with four models found
\end{abstract}

Calle Francisco Villa 450, Col. Dr. Miguel Silva, Morelia, Michoacán, México. Tel.: (52) 14433958130. fbetanzos@umich.mx 
in the different adaptations to Spanish; in the second phase, models that analyze acquiescence are taken into account, and in the third phase, a cross-validation of those models with a clinical population is made. The results indicate that the scale is one-dimensional both in the case of clinical samples $(\chi 2=154.84, \mathrm{gl}=135, \mathrm{p}<0.001, \mathrm{CFI}=0.99$, TLI $=0.99$, RMSEA $=0.03$ ), as well as in the general population. However, for the latter, a method factor was added for the treatment of acquiescence $\left(\chi^{2}=252.14, \mathrm{gl}=134, p<0.001, \mathrm{CFI}=0.95, \mathrm{TLI}=0.94, \mathrm{RMSEA}=0.03\right)$. The results show the importance of using analyzes and models that consider the nature of the data and the characteristics of the sample to provide more solid evidence for construct validity.

Keywords: adaptation, hopelessness, validity, suicidal risk, acquiescence.

\section{Introducción}

En 1967, Aaron Beck, el psiquiatra estadounidense, planteó que la desesperanza es uno de los elementos de la triada cognitiva de la depresión y de los síntomas depresivos (Beck et al., 1974); en estudios recientes, esta misma se considera un factor determinante en el estudio de las causas de la depresión (González et al., 2018; Vives \& Dueñas, 2018; Waszczuk et al., 2016), así como un indicador poderoso de la ideación e intento suicida en muestras clínicas (Beck et al., 1974; Gheihman et al., 2016; Klonsky et al., 2012; Nissim et al., 2010; Steer et al., 1993; Troister et al., 2015; Wang et al., 2015) y no clínicas (Aliaga et al., 2006; Horwitz et al., 2016; Mikulic et al., 2009; Mitchell et al., 2016; Ribeiro et al., 2015; Suárez-Colorado et al., 2019).

Específicamente, Beck et al. (1974) señalan que la desesperanza no es un estado emocional difuso, vago y difícil de cuantificar en estudios científicos, sino que, por el contrario, es un constructo que hace referencia a un sistema organizado de expectativas negativas sobre el propio futuro y la propia persona. A partir de esto, dichos autores desarrollaron la Escala de Desesperanza (BHS, por sus siglas en inglés), con el propósito de proporcionar a la comunidad científica y clínica una herramienta confiable, sensible y fácil de utilizar para evaluar el estado de desesperanza.

La BHS es una escala dicotómica que ha demostrado propiedades psicométricas adecuadas en varios países (Hanna et al., 2011; Madeira et al., 2011; Mystakidou et al., 2008). Incluso, en la versión en español se han hecho diversas traducciones y adaptaciones en países hispanoamericanos, como España, Perú, Colombia, Argentina y México, en los que se confirma su utilidad como uno de los instrumentos más generalizados en el ámbito clínico para el tamizaje de la depresión y el riesgo suicida (Aliaga et al., 2006; Bobes et al., 2002; Córdova \& Rosales, 2011; González, 2009; Mikulic et al., 2009). Sin embargo, en dichos trabajos no se ha logrado replicar la estructura factorial original, $\mathrm{ni}$ la que se ha propuesto recientemente para la adaptación a otros países (Innamorati et al., 2014; Kocalevent et al., 2017; Steer et al., 1997).
Es importante tener en cuenta que las estructuras factoriales descritas varían según el tipo de muestra (p. ej., si son muestras clínicas o no clínicas) y el procedimiento utilizado para el análisis factorial (Beck \& Steer, 1993; Boduszek \& Dhingra, 2016; Steer et al., 1997). Por ejemplo, en el estudio original, Beck et al. (1974) aplicaron la escala a población psiquiátrica hospitalizada por intento de suicidio, y para la validez de constructo llevaron a cabo un análisis factorial exploratorio (AFE) con componentes principales como método de extracción de factores y rotación varimax; en los resultados, los autores identificaron tres factores: al primer factor lo denominaron Feelings About the Future (sentimientos acerca del futuro), el segundo factor, Loss of Motivation (pérdida de la motivación), y al tercer factor, Future Expectations (expectativas sobre el futuro).

En el caso de las adaptaciones al español, por ejemplo en Argentina, Mikulic et al. (2009) aplicaron la escala adaptada a 377 personas de población general, y sus resultados dieron cuenta de una confiabilidad de .78 , así como de la presencia de tres factores que se agrupan de forma distinta al estudio original.

En Perú, Aliaga et al. (2006) llevaron a cabo un estudio con muestras no clínicas, clínicas y médicas - personas con intento de suicidio, personas con depresión, hipertensos, personas que podían padecer asma o tuberculosis, personas con esquizofrenia, consumidores de cocaína y población general-, donde encontraron un alfa moderado (.80), seis factores y sensibilidad para la identificación de personas con depresión e intento de suicidio.

En Colombia, González (2009) utilizó la traducción española del banco de instrumentos básicos para la práctica clínica (Bobes et al., 2002), la aplicó a 543 participantes, en su mayoría estudiantes de psicología, y el análisis de las propiedades psicométricas mostró una fiabilidad moderada (.83), así como cinco dimensiones distintas a partir del análisis factorial.

Y en México, la escala se ha aplicado a pacientes de hospitales psiquiátricos con intento suicida (Almeida-Montes et al., 2000; Ibarra et al., 2000; Quintanilla et al., 2003), a pacientes hospitalizados que padecían alguna enfermedad 
150

o condición psiquiátrica (Espinosa et al., 2009), a personas que acudieron a consulta médica externa (Borges et al., 2000; Mondragón et al., 1998; Satorres et al., 2018), y a estudiantes (Córdoba \& Rosales, 2011; Lazarevich, Delgadillo \& Rodríguez, 2009). De estos, destaca la investigación de Córdoba y Rosales (2011) debido a que el análisis factorial arrojó tres dimensiones, una de las cuales coincidió con el factor de sentimientos sobre el futuro propuesto por Beck et al. (1974) —aunque no ocurrió lo mismo con los otros dos factores-.

Es importante señalar que, hasta el momento, los estudios de validez factorial de la BHS de las versiones en español han utilizado el análisis factorial exploratorio con componentes principales como método de extracción de factores, aun cuando este método se considera poco recomendable para escalas dicotómicas (Choi et al., 2010; Freiberg et al., 2013); además, no han probado la estructura interna mediante análisis factorial confirmatorio; y no han hecho comparaciones para establecer si la estructura factorial de la escala es la misma en población no clínica que en personas que han tenido intento de suicidio - esto para identificar si hay equivalencia en la estructura de la escala entre estas dos condiciones-.

Actualmente, en otros países se debate sobre si la BHS es unidimensional o multidimensional (Innamorati et al., 2014; Pompili et al., 2007; Steer et al., 1997), y, en los últimos años, la discusión se ha enfocado en la preocupación de si existe una estructura bifactorial de optimismo/ pesimismo, en la que el optimismo se evaluaría con los ítems que se construyeron con una visión positiva (p. ej., "Veo el futuro con esperanza y entusiasmo"), mientras que el pesimismo se mediría con los que se construyeron con una visión negativa (p. ej., "El futuro me parece vago e incierto"). Por el contrario, la visión unidimensional concibe que estas dos fases son en realidad la medida de un único constructo sustantivo de la psicopatología: la desesperanza.

Ahora bien, debe tenerse en cuenta que la validez de constructo es particularmente difícil para escalas balanceadas, como es el caso de la BHS, ya que estas escalas están diseñadas para evitar la aquiescencia y tienen el mismo número o aproximadamente el mismo número de ítems directos $\mathrm{e}$ inversos, de modo que, para estos casos, los ítems tienden a agruparse a partir de su contenido semántico (Vautier \& Pohl, 2009; Vautier et al., 2004). De hecho, en el análisis factorial exploratorio de escalas balanceadas surgen factores que no están basados en la dimensión teórica, sino en la polaridad del ítem - a lo que se conoce como factor de Método-(Savalei \& Falk, 2014), lo cual significa que la estructura bifactorial de la BHS podría ser causa del método empleado (Innamorati et al., 2014).

Dado que el cuestionario es ampliamente utilizado para medir desesperanza en situaciones de riesgo de suicidio, los aspectos mencionados tienen implicaciones clínicas importantes, y es por ello que el objetivo del presente estudio fue investigar la estructura interna de la de la Escala de Desesperanza de Beck en dos muestras, una de estudiantes universitarios y otra de jóvenes con intento de suicidio de alta letalidad, para luego comparar los modelos propuestos en este estudio y en las adaptaciones al español con el modelo original, así como con modelos alternativos que tomen en cuenta la naturaleza de la escala y de las muestras estudiadas.

Cabe mencionar que el análisis de la estructura interna se realiza utilizando el análisis factorial confirmatorio mediante modelos de ecuaciones estructurales con un estimador robusto para ítems dicotómicos con distribución no normal, que es lo apropiado en este tipo de escalas; y que, además, en esta investigación se realizan tres análisis: primero se compara el ajuste de todos los modelos propuestos de las diferentes traducciones al español en relación con la estructura original propuesta por Beck et al. (1974) en una muestra de estudiantes universitarios; segundo, se analizan los modelos que se han sugerido para el análisis de la aquiescencia —en este caso, se compara un modelo que contiene un factor de método con dos modelos básicos, el modelo de un factor general (desesperanza) y el modelo de dos factores correlacionados (optimismo/pesimismo) - ; y tercero, se aplican estos modelos a una muestra de personas que han tenido algún intento de suicidio, para observar el ajuste en esta muestra en particular.

\section{Método}

\section{Participantes}

La muestra total fue de 1410 participantes que provienen de dos muestras: la muestra "A", conformada por 1260 estudiantes de pregrado de la Universidad Autónoma de Aguascalientes, México (721 mujeres y 539 hombres), elegidos mediante un muestreo no probabilístico de ocho de los diez centros académicos, con un rango de edad que osciló entre los 17 y los 30 años $(M=19.84, D E=1.94)$; y la muestra "B", que corresponde a 150 sujetos (63 mujeres y 87 hombres), cuyo rango de edad estuvo entre los 14 y los 37 años $(M=23.1, D E=6.2)$, reportados y atendidos 
por intento de suicidio de alta letalidad por el servicio de emergencias 911 del Estado de Aguascalientes.

\section{Diseño}

Se empleó un diseño no experimental de tipo transversal (una única medida) para un análisis correlacional multivariado, necesario para los análisis factoriales y comparaciones de grupo.

\section{Instrumento}

Se utilizó la Escala de Desesperanza de Beck, desarrollada por Beck et al. (1974), una escala de 20 ítems que evalúa las actitudes negativas sobre el futuro, que mostró una fiabilidad de .93 en el artículo original. En su diseño, los autores consideraron veinte ítems en total, once que refieren a enunciados pesimistas y nueve a enunciados optimistas sobre el futuro, con opciones de respuesta de "falso" o "verdadero". De este modo, las puntuaciones van de 0 a 20, donde una mayor puntuación indica una mayor desesperanza. Los índices de confiabilidad del instrumento en español, medidos con el alfa de Cronbach, han mostrado algunas variaciones: $\alpha=.78$ en el estudio de Mikulic et al. (2009); $\alpha=.80$ en el de Aliaga et al. (2006); y $\alpha=.83$ en el de González (2009).

La adaptación al español que fue utilizada en el presente estudio se realizó teniendo en cuenta los procedimientos estándares de traducción inversa (Eremenco et al., 2005; Van de Vijver \& Tanzer, 2004), de modo que, para la adaptación del instrumento - en la que se empleó la versión original en inglés de la Escala de Desesperanza de Beck (la BHS, de Beck et al., 1974)—, se realizó una primera traducción del inglés al español — por parte de cuatro profesionales bilingües-; posteriormente, se tradujo la escala de español a inglés - por un traductor experto-; y a continuación se hicieron comparaciones entre la versión original y la versión traducida hasta que no se encontraron diferencias relevantes. Después, se realizó una adaptación cultural en la que jueces expertos debían analizar la claridad de las instrucciones y de los reactivos, para así eliminar problemas de usos lingüísticos culturales. A esta adaptación se le denominó BHS-UAA.

\section{Procedimiento}

Para la obtención de la primera muestra, es decir, los 1260 estudiantes universitarios, se realizó un muestreo no probabilístico de un total de 25 programas educativos de pregrado de la Universidad Autónoma de Aguascalientes, en México. Después, para la evaluación, primero se contactó a las autoridades correspondientes - incluyendo a los profesores - para obtener el consentimiento informado de cada uno de los centros académicos, así como la autorización expresa de los profesores para utilizar su espacio y tiempo de clase durante la evaluación; posteriormente, se contactó a los alumnos en sus aulas durante su horario de clase - acordado con el profesor-, y se les informó sobre los objetivos y fines del estudio, así como la forma en que sería tratada la información recabada; finalmente, tras obtener el consentimiento informado de los estudiantes, se procedió a aplicarles el instrumento en cuestión.

Por otra parte, la muestra clínica se eligió de manera no probabilística y estuvo conformada por 150 jóvenes, de los cuales el $42.7 \%$ eran hombres $(n=64)$, y $57.3 \%$, mujeres $(n=86)$, con edades entre los 14 y los 35 años, con una media de 23.07 años $(D E=6.19)$, que fueron atendidos por intento de suicidio de alto riesgo y que aceptaron que se les realizara un seguimiento por el sistema de salud del Estado. Las llamadas fueron captadas por el servicio telefónico de emergencias 911 del Centro Estatal de Telecomunicaciones $\mathrm{C} 4$, y los participantes se eligieron de la base de datos de personas que participaron en el estudio denominado Modelo Diagnóstico para la Prevención del Suicidio en Adolescentes y Jóvenes del Estado de Aguascalientes (UAA PIPS 14-3N).

\section{Análisis de datos}

Se realizó un Análisis Factorial Confirmatorio (AFC) para los diferentes modelos propuestos en las adaptaciones al español descritas previamente (véase Tabla 1). Específicamente, para el AFC se utilizó el estimador Mínimos Cuadrados Ponderados Robustos (WLSMV, por sus siglas en inglés), el cual no asume que las variables deban estar distribuidas normalmente, el método más apropiado para los AFC con datos dicotómicos (Muthén \& Muthén, 2006); y, para el análisis del ajuste de los diferentes modelos propuestos por los autores, se tomaron en cuenta diversos indicadores, a saber: (a) el estadístico chi-cuadrado $\left(\chi^{2}\right)$, (b) el índice de ajuste comparativo (CFI), (c) el índice de Tucker Lewis (TLI), (d) la raíz cuadrada del error medio de aproximación (RMSEA), y (e) el $90 \%$ de intervalo de confianza para dicho indicador $(90 \% \mathrm{CI})$. Un ajuste aceptable del modelo se define por los siguientes criterios: RMSEA < .08 (90\% CI), CFI > .90, TLI > .90 (Bentler, 1990; Hu \& Bentler, 1999); considerando un buen ajuste si el RMSEA < .05, CFI > .95, TLI > .95 (Bentler, 1990; Hu \& Bentler, 1999). Los análisis se realizaron mediante el programa MPlus 7.1 (Muthén \& Muthén, 2012). 
Tabla 1.

\begin{tabular}{|c|c|c|c|c|c|}
\hline & $\begin{array}{l}\text { Beck et al. } \\
\text { (1974) }\end{array}$ & $\begin{array}{l}\text { Mikulic et al. } \\
\quad(2009)\end{array}$ & $\begin{array}{l}\text { Aliaga et al. } \\
(2006)\end{array}$ & $\begin{array}{l}\text { González } \\
\text { (2009) }\end{array}$ & $\begin{array}{c}\text { Córdoba \& Rosales } \\
(2011)\end{array}$ \\
\hline Alfa de Cronbach & 0.93 & 0.78 & 0.8 & 0.83 & 0.78 \\
\hline $\begin{array}{c}\text { Número de factores } \\
\text { extraídos }\end{array}$ & 3 & 3 & 6 & 5 & 3 \\
\hline \multicolumn{6}{|c|}{ Ítems por factor } \\
\hline Factor 1 & $\begin{array}{c}1,5,6 \\
13,15 \text { y } 19\end{array}$ & $\begin{array}{l}3,4,6 \\
13 \text { y } 19\end{array}$ & $\begin{array}{l}6,7,9,12 \\
16,17 \text { y } 20\end{array}$ & $\begin{array}{l}1,3,8,10 \\
13,15 \text { y } 19\end{array}$ & $\begin{array}{l}1,6,13 \\
15 \text { y } 19\end{array}$ \\
\hline Factor 2 & $\begin{array}{c}2,3,9,11 \\
12,16,17 \text { y } 20\end{array}$ & $\begin{array}{l}2,7,9,11,12 \\
14,16,17 \text { y } 20\end{array}$ & $\begin{array}{l}5,10 \\
14 \text { y } 15\end{array}$ & $\begin{array}{c}11,12,13,14 \\
16,1 \text { y } 8\end{array}$ & $\begin{array}{c}2,3,9,11 \\
12,16,17 \text { y } 20\end{array}$ \\
\hline Factor 3 & $\begin{array}{c}4,7,8 \\
14,10 \text { y } 18\end{array}$ & $\begin{array}{c}1,5,8 \\
10,15 \text { y } 18\end{array}$ & 13 у 19 & $\begin{array}{l}2,4 \\
9 \text { y } 14\end{array}$ & $\begin{array}{l}4,7,8 \\
14 \text { y } 18\end{array}$ \\
\hline Factor 4 & & & 1,4 y 18 & $\begin{array}{l}2,4 \\
9 \text { y } 14\end{array}$ & \\
\hline Factor 5 & & & 3 & 5 у 6 & \\
\hline Factor 6 & & & 8 & & \\
\hline
\end{tabular}

\section{Resultados}

Los datos que permiten la comparación de modelos se presentan en la Tabla 2, en la cual se pueden encontrar cuatro apartados: en los primeros tres se trabaja con la muestra "A", y en el último, con la muestra "B". En el primer apartado se ajusta el modelo original de Beck et al., (1974) de tres factores; en el segundo se muestran los modelos de las adaptaciones al español, donde es de destacar que en algunos casos no se alcanzó una solución viable, dado que la matriz de covarianza de la variable latente no está definida de manera positiva - en estos modelos se antepone un asterisco para su identificación-; en el tercero se presentan tres modelos: (a) el unidimensional propuesto con muestras clínicas (Mystakidou et al., 2008), (b) el de dos factores correlacionados —optimismo/pesimismo(Nissim et al., 2010), y (c) el unidimensional con un factor general para la aquiescencia (Savalei \& Falk, 2014) —el cual intenta capturar las tendencias individuales de usar las categorías de respuesta de forma consistente a través de los ítems, pero de manera idiosincrática entre los individuos (Abad et al., 2016) - ; y, por último, en el cuarto apartado se hace una validación cruzada de los modelos del tercer apartado con los datos de la muestra "B" - una muestra clínica-

En general, los índices de ajuste señalan que el modelo original tiene un ajuste aceptable $(\chi 2=373.713, \mathrm{gl}=167$, $\mathrm{p}<0.001, \mathrm{CFI}=0.92, \mathrm{TLI}=0.91, \mathrm{RMSEA}=0.04)$, mejor que el encontrado en los modelos de las adaptaciones al español previas a la propuesta en esta investigación. Sin embargo, en la muestra "A" (de estudiantes universitarios) el mejor modelo es el tercero, el unidimensional, en el que se propone un factor de método para el tratamiento de la aquiescencia $\left(\chi^{2}=252.14\right.$, $\mathrm{gl}=134, \mathrm{p}<0.001, \mathrm{CFI}=0.95, \mathrm{TLI}=0.94, \mathrm{RMSEA}=0.03$ ).
Estos mismos modelos aplicados a la muestra "B" (de personas con intento de suicidio) muestran que el instrumento es unidimensional (primer modelo), y que el modelo de dos factores y el modelo para el tratamiento de la aquiescencia muestran dependencia lineal entre los factores, razón por la cual la solución es inadmisible. En este caso, se observa un ajuste excelente en el modelo unidimensional para la muestra "B" $\left(\chi^{2}=154.84, \mathrm{gl}=135, \mathrm{p}>0.05, \mathrm{CFI}=0.99\right.$, $\mathrm{TLI}=0.99$, RMSEA $=0.03)$.

Es importante notar que tanto el ítem 5 - "Tengo el tiempo suficiente para lograr las cosas que más quiero hacer"- como el ítem 11 — "Todo lo que puedo ver en el futuro es más desagradable que agradable"- presentaron pesos factoriales no significativos $(p>0.05)$ en todos los modelos, por lo cual se decidió eliminarlos.

Por otra parte, en la Tabla 3 se presentan los pesos factoriales $(\lambda)$ sobre el factor general denominado "Desesperanza". Acá, los ítems están organizados de mayor a menor peso factorial en la muestra "B", que es donde este modelo presenta mayor ajuste; en el caso de la muestra " $\mathrm{B}$ ", el ítem cuatro tiene un peso factorial bajo $(\lambda=0.24)$, sin embargo, para la muestra "A" este mismo tiene un peso factorial aceptable $(\lambda=0.48)$; además, todos los ítems con pesos factoriales negativos (6, $1,15,13,19,8,10$ y 3 ) miden, en el caso del modelo de dos dimensiones, la dimensión llamada optimismo — donde el análisis de fiabilidad mediante el estadístico de KuderRichardson mostró una fiabilidad buena (KR-20 = .916)-, mientras que los ítems con una visión negativa $(2,12,7,20$, $9,16,18,17,14$ y 4) miden la dimensión denominada pesimismo - con un buen nivel de fiabilidad — $(\mathrm{KR}-20=.926)$; $\mathrm{y}$ en el caso del modelo unidimensional, la fiabilidad total de la escala fue alta $(K R-20=.948)$. 
Tabla 2.

Índices de ajuste de los modelos para el BHS

\begin{tabular}{|c|c|c|c|c|c|c|}
\hline Modelos & $\chi^{2}$ & $g l$ & $\mathrm{p}$ & CFI & TLI & RMSEA $(90 \% \mathrm{CI})$ \\
\hline \multicolumn{7}{|c|}{ 1. Modelo original. Muestra " $A$ ". } \\
\hline Beck et al. (1974). & 373.713 & 167 & $<0.001$ & 0.92 & 0.91 & $0.04(.034, .045)$ \\
\hline \multicolumn{7}{|c|}{ 2. Adaptaciones al español. Muestra "A". } \\
\hline Mikulic et al. (2009) & 396.92 & 167 & $<0.001$ & 0.91 & 0.9 & $0.04(.037, .047)$ \\
\hline
\end{tabular}

*Aliaga et al. (2006).

La matriz de covarianza de la variable latente de no está definida de manera positiva.

*Gonzalez (2009).

$\begin{array}{lllllll}\text { Córdoba y Rosales (2011). } & 656.95 & 169 & <0.001 & 0.819 & 0.797 & 0.06(.056, .066)\end{array}$

3. Modelos para el tratamiento de la aquiescencia. Muestra " $A$ ".

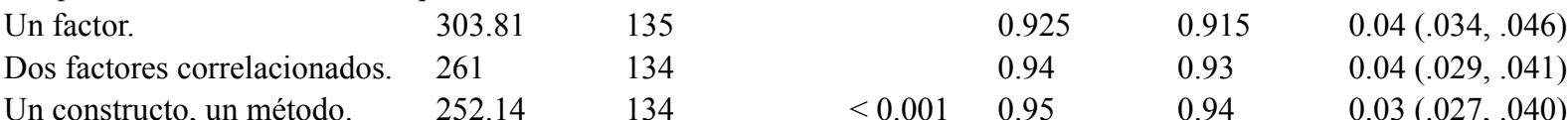

4. Modelos para el tratamiento de la aquiescencia. Muestra " $B$ ".

$\begin{array}{lllllll}\text { Un factor. } & 154.84 & 135 & >0.05 & 0.99 & 0.99 & 0.03(.001, .005)\end{array}$

*Dos factores correlacionados. La matriz de covarianza de la variable latente de no está definida de manera positiva. *Un constructo, un método.

Nota. En estos modelos no se consideran los ítems 5 y $11 . \chi^{2}=$ chi-cuadrada; $\mathrm{gl}=$ grados de libertad; $\mathrm{p}=$ significancia estadística; $\chi^{2} /$ $\mathrm{gl}=$ chi-cuadrada dividida por grados de libertad; $\mathrm{CFI}=$ índice de ajuste comparativo; TLI = índice de Tucker Lewis; RMSEA= error cuadrático medio de aproximación; $\mathrm{CI}=$ intervalo de confianza. * No se ha alcanzado una solución viable dado que la matriz de covarianza de la variable latente no está definida de manera positiva.

Tabla 3.

Estimaciones estandarizadas mediante el método de mínimos cuadrados ponderados robustos (WLSMV)

\begin{tabular}{|c|c|c|c|c|c|c|c|}
\hline \multirow{2}{*}{\multicolumn{2}{|c|}{ Ítem }} & \multicolumn{3}{|c|}{ Muestra "A" } & \multicolumn{3}{|c|}{ Muestra "B" } \\
\hline & & $\lambda$ & SE & $R^{2}$ & $\lambda$ & SE & $R^{2}$ \\
\hline 6 & En el futuro espero triunfar en las cosas que más me interesan. & -0.82 & 0.073 & 0.736 & -0.99 & 0.025 & 0.992 \\
\hline 1 & Veo el futuro con esperanza y entusiasmo. & -0.79 & 0.046 & 0.690 & -0.93 & 0.031 & 0.857 \\
\hline 15 & Tengo fe en el futuro. & -0.74 & 0.040 & 0.620 & -0.91 & 0.040 & 0.821 \\
\hline 13 & $\begin{array}{l}\text { Cuando veo hacia el futuro tengo la esperanza de ser más feliz } \\
\text { que ahora. }\end{array}$ & -0.54 & 0.060 & 0.337 & -0.83 & 0.060 & 0.693 \\
\hline 19 & Puedo esperar más tiempos buenos que malos. & -0.70 & 0.048 & 0.549 & -0.64 & 0.091 & 0.410 \\
\hline 8 & $\begin{array}{l}\text { Espero recibir más cosas buenas de la vida que la mayoría de las } \\
\text { personas. }\end{array}$ & -0.51 & 0.051 & 0.320 & -0.60 & 0.081 & 0.361 \\
\hline 10 & Mis experiencias me han preparado para el futuro. & -0.52 & 0.066 & 0.330 & -0.57 & 0.094 & 0.320 \\
\hline 3 & $\begin{array}{l}\text { Cuando las cosas van mal pienso que no pueden quedarse así } \\
\text { siempre. }\end{array}$ & -0.50 & 0.060 & 0.304 & -0.34 & 0.112 & 0.115 \\
\hline 2 & $\begin{array}{l}\text { Podría darme por vencido porque no puedo hacer que las cosas } \\
\text { sean mejores para mí. }\end{array}$ & 0.45 & 0.065 & 0.239 & 0.82 & 0.05 & 0.671 \\
\hline 12 & No espero obtener lo que realmente quiero. & 0.79 & 0.039 & 0.653 & 0.81 & 0.059 & 0.653 \\
\hline 7 & Mi futuro me parece muy oscuro. & 0.80 & 0.056 & 0.666 & 0.81 & 0.056 & 0.649 \\
\hline 20 & Es inútil tratar de conseguir algo porque no lo conseguiría. & 0.82 & 0.05 & 0.696 & 0.80 & 0.054 & 0.636 \\
\hline 9 & No tengo suerte ni razón para creer que la tendré en el futuro. & 0.83 & 0.046 & 0.721 & 0.73 & 0.066 & 0.535 \\
\hline 16 & No deseo algo porque nunca consigo lo que quiero. & 0.81 & 0.048 & 0.681 & 0.71 & 0.068 & 0.497 \\
\hline 18 & El futuro me parece vago e incierto. & 0.63 & 0.040 & 0.440 & 0.68 & 0.074 & 0.460 \\
\hline 17 & Es muy poco probable que el futuro tenga una satisfacción real. & 0.57 & 0.059 & 0.353 & 0.56 & 0.088 & 0.311 \\
\hline 14 & Las cosas no funcionan como me gustaría. & 0.52 & 0.051 & 0.303 & 0.49 & 0.089 & 0.243 \\
\hline 4 & No puedo imaginar mi vida dentro de 10 años. & 0.48 & 0.052 & 0.259 & 0.24 & 0.105 & 0.055 \\
\hline
\end{tabular}

Nota. $\lambda=$ peso factorial estandarizado, $\mathrm{SE}=$ error estándar, $R^{2}=$ tamaño del efecto. 
Finalmente, en la Figura 1 se muestran los modelos con mayor ajuste en cada una de las muestras. Específicamente, la Figura 1a corresponde a la muestra "A" (estudiantes universitarios), donde los pesos factoriales en el factor de Método son de 0.21 para los ítems positivos, y de -0.21 para los ítems negativos, mientras que los pesos factoriales estandarizados de la figura en el factor llamado "Desesperanza" son los que se presentan en la Tabla 3 para la muestra correspondiente. Por otra parte, la Figura $1 \mathrm{~b}$ representa el modelo unidimensional para la muestra clínica.

\section{Discusión}

La Escala de Desesperanza de Beck es uno de los instrumentos más utilizados en el ámbito clínico por su relevancia en el estudio y tratamiento de la depresión, así como por su capacidad para predecir la ideación y el intento de suicidio. Como se expuso, esta es una de las razones por las cuales este instrumento ha sido adaptado en una gran variedad de países (Hanna et al., 2011; Madeira et al., 2011; Mystakidou et al., 2008); sin dejar de mencionar, por supuesto, las adaptaciones que se han hecho para población hispanohablante, incluida la población mexicana (Aliaga et al., 2006; Bobes et al., 2002; Córdoba \& Rosales, 2011; González, 2009; Mikulic et al., 2009).

El propósito de analizar la validez factorial de la Escala de Desesperanza de Beck (BHS-UAA) y compararla con muestras clínicas y no clínicas ha respondido a la intención de obtener más datos acerca de su sensibilidad, confiabilidad y validez, lo cual se ha podido lograr al comparar los diferentes modelos de medida propuestos hasta el momento

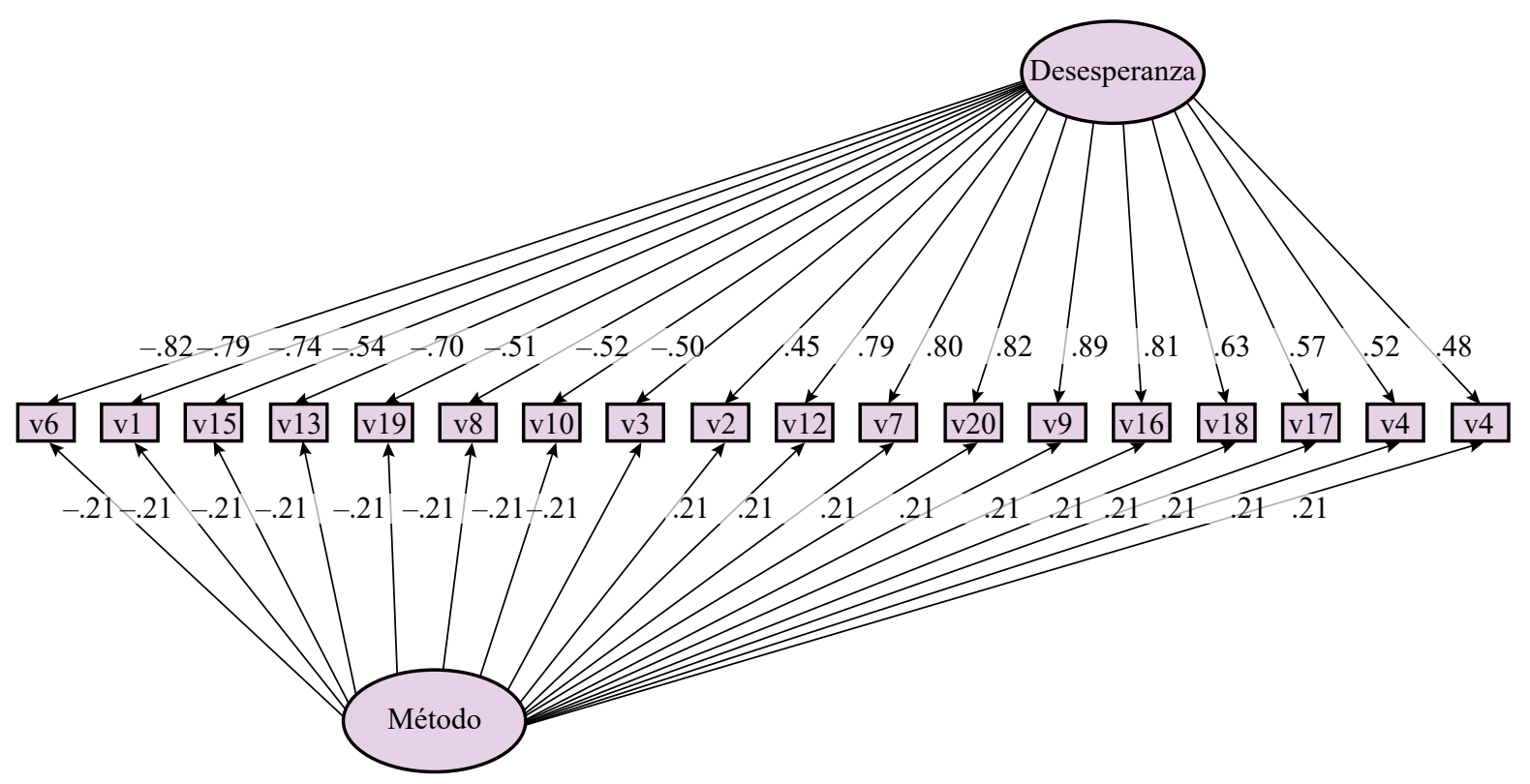

Figura 1a. Diagrama del modelo con mayor ajuste en la muestra de estudiantes universitarios.

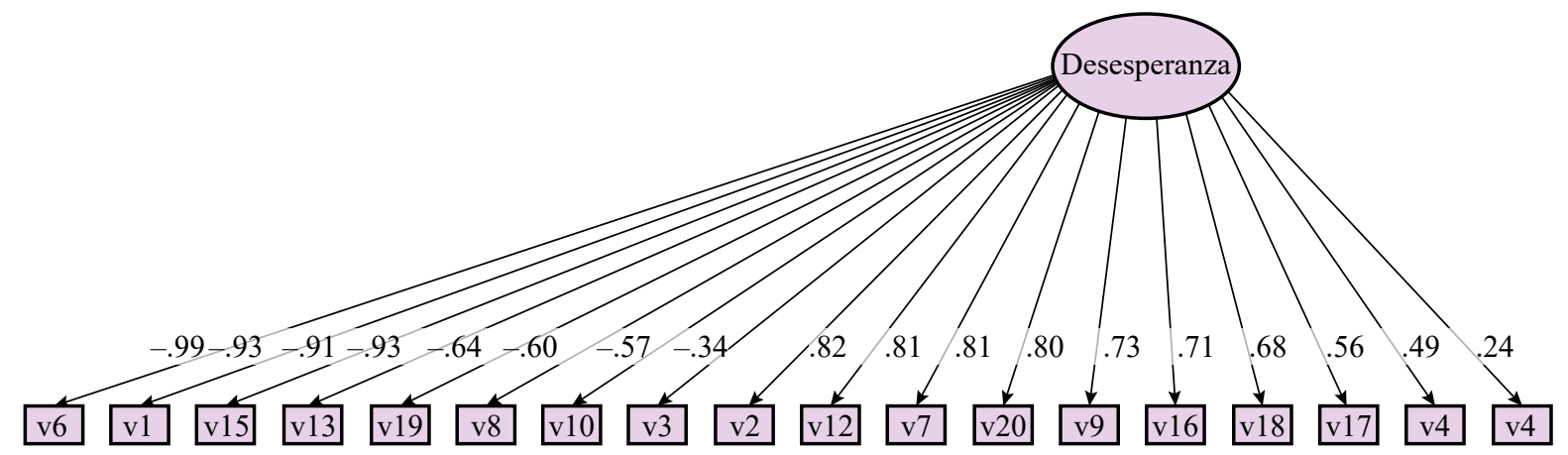

Figura $1 b$. Diagrama del modelo con mayor ajuste en la muestra clínica. 
en las adaptaciones al español y su relación con el modelo propuesto por Beck et al. (1974), así como al realizar pruebas con modelos que tomen en cuenta la aquiescencia. Teniendo esto en cuenta, la preocupación fundamental del estudio es reducir al máximo el error de medida y el error de análisis de consistencia y validez de constructo, para así contar con un instrumento con grandes alcances psicométricos que, a la vez, sea confiable en población que esté en riesgo de suicidio.

De este modo, a partir de los análisis realizados en el presente estudio, la BHS-UAA mostró una alta consistencia interna en las dos poblaciones estudiadas, $\mathrm{y}$, de igual forma, resultó ser sensible y discriminante en población con intento de suicidio de alta letalidad, por lo que demuestra ser un instrumento de gran utilidad en la detección de la desesperanza. Además, estos datos son consistentes con hallazgos reportados en las adaptaciones realizadas en diversos países del mundo, y en conjunto ofrecen un fuerte soporte empírico sobre el adecuado funcionamiento psicométrico de la escala. Sin embargo, la evidencia reportada también exhibe la existencia de múltiples conformaciones factoriales de la escala, susceptibles a la población de estudio, y, de hecho, es sabido que los métodos de análisis factorial clásicos que parten de la matriz de correlaciones de Pearson tienden a sobredimensionar el número de factores cuando los datos son dicotómicos, especialmente cuando hay pocos ítems por factor o cuando los pesos factoriales son pequeños, como ocurre en este caso (Fava \& Velicer, 1992; Garrido et al., 2011).

En el presente análisis se tomaron en cuenta estas consideraciones para proponer un método de estimación que parte de la matriz de correlaciones tetracóricas, además de que se prueban los modelos de las adaptaciones en español de la escala, a partir del cual los resultados señalan que las versiones de tres factores son mejores que aquellas que proponen más de tres factores - incluso, no se logró un ajuste de las versiones con cinco o seis factores, dado que la matriz de covarianzas de las variables latentes no está definida de manera positiva- En un análisis más profundo, se encuentran correlaciones mayores a uno entre dos factores, lo que se puede considerar como estimaciones que están fuera de los rangos admisibles y como un signo de que el modelo es incorrecto. De este modo, se puede afirmar que en la comparación entre el modelo original y las adaptaciones al español los resultados señalan que el mejor ajuste se obtiene con una estructura factorial similar a la propuesta original desarrollada por Beck et al. (1974).

Adicional a esto, en el análisis de los distintos factores que se han reportado en la escala de desesperanza es importante resaltar dos elementos: el primero, que la BHS-UAA es una adaptación realizada en estricto apego al método back-translation (por su denominación en inglés), donde se ha tomado como fuente principal la escala desarrollada por Beck et al. (1974), razón por la cual se trata de una versión que se apega fielmente a los principios de la escala original, atendiendo íntegramente al significado y contenido de los ítems originales en la adaptación cultural, y solventando así los problemas observados en las demás adaptaciones al español; y el segundo, que refiere al tipo y organización de ítems y de respuestas que tiene el instrumento, pues la BHS-UAA, al igual que la BHS de Beck et al. (1974), es una escala balanceada con ítems directos e inversos, lo cual disminuye y controla la posible tendencia de respuesta en aquellos sujetos que responden afirmativamente a los ítems independientemente de su contenido - lo cual quiere decir que esta versión controla el factor invalidante que supone la aquiescencia - . No obstante, esta última característica dificulta el análisis de validez de constructo, ya que los resultados reflejan más la polaridad de los ítems que la dimensión teórica del constructo, que en este caso es la desesperanza (Boduszek \& Dhingra, 2016; Innamorati et al., 2014; Vautier et al., 2004).

En este contexto, los resultados con los datos de los universitarios demuestran que un modelo unidimensional en el que se toma en cuenta la aquiescencia es el que obtiene mejores indicadores de ajuste, y que, por el contrario, en la muestra de personas con intento de suicidio, a quienes se administró el instrumento mediante entrevista, se encuentra un mejor ajuste en el modelo unidimensional sin el factor de aquiescencia - de hecho, los dos modelos en los que se considera la aquiescencia no pueden estimarse-. Esto es consistente con la teoría, pues se sabe que el problema de la aquiescencia se observa especialmente en muestras de población normal, donde es más probable que se muestren este tipo de tendencias de respuesta, mientras que cuando la aplicación la realiza un profesional esta tendencia desaparece.

Tomando en cuenta lo anterior, se puede afirmar que el método empleado para analizar la estructura interna del BHS-UAA es adecuado debido a que considera la utilización de estimadores robustos de ecuaciones estructurales para ítems dicotómicos que no se distribuyen de forma normal. Asimismo, al haber sometido estos resultados a una comparación de ajuste con los factores reportados en las distintas adaptaciones realizadas al español y con la propuesta original de Beck et al. (1974), y gracias a la comparación con los modelos surgidos para el análisis de la aquiescencia - que se aplican tanto a personas con intento de suicidio de alta letalidad como a personas de la población general-, es posible concluir que la estructura del BHS-UAA tiene, hasta el momento, una mayor solidez que el resto de versiones analizadas. 
156

Finalmente, cabe mencionar que los resultados del presente estudio tienen al menos dos implicaciones prácticas: primero, que se aporta evidencia relacionada con la unidimensionalidad de la escala, por lo cual el cálculo de tres o más puntuaciones en diferentes factores no tiene mayor sentido; $\mathrm{y}$, segundo, que la aplicación en contextos clínicos hecha por profesionales minimiza la probabilidad de errores debidos a la aquiescencia (Meisenberg \& Williams, 2008). Sin embargo, es importante señalar que el presente estudio no considera muestras clínicas en las que existan bajos niveles de motivación para ser evaluados, como, por ejemplo, en población psiquiátrica, en población mayor (Tovar et al., 2019), en enfermos crónicos, en personas con orientación sexual diversa (Avendaño-Prieto et al., 2019), o en personas que consumen drogas, por lo cual se recomienda para futuras investigaciones incluir este tipo de poblaciones.

\section{Referencias}

Abad, J., Sorrel, M. A., Garcia, L. F., \& Aluja, A. (2016). Modeling General, Specific, and Method Variance in Personality Measures: Results for ZKA-PQ and NEO-PI-R. Assessment, 19(1), 1-19. https:/doi. org/10.1177/1073191116667547

Aliaga, T. J., Rodríguez, R. L., Ponce, D. C., Frisancho, L. A., \& Enríquez, V. J. (2006). Escala de Desesperanza de Beck (BHS): Adaptación y Características Psicométricas. Revista de Investigación en Psicología. 9(1), 69-79. http://ateneo. unmsm.edu.pe/handle/123456789/1563?show=full

Almeida-Montes, L. G., Valles-Sánchez, V., Moreno-Aguilar, J., Chávez-Balderas, R. A., García-Marín, J. A., Cortés, S. J., \& Hheinze-Martin, G. (2000). Relation of serum cholesterol, lipid, serotonin and tryptophan levels to severity of depression and to suicide attempts. Journal of Psychiatry \& Neuroscience, 25(4), 371-377. https://www.ncbi.nlm.nih. gov/pmc/articles/PMC1407732/?page $=1$

Avendaño-Prieto, B. L., Betancort, M., Bernal-Aguirre, A., González-Martínez, L. A., Gómez-Sánchez, S. M., \& Villalobos-Sánchez, C. F. (2019). Celos, desesperanza e ideación suicida en población con orientación sexual diversa. Universitas Psychologica, 18(4), 1-12. https://doi. org/10.11144/Javeriana.upsy18-4.cdis

Beck, A., Weissman, A., Lester, D., \& Trexler, L. (1974). The Measurement of Pessimism: The Hopelessness Scale. Journal of Consulting and Clinical Psychology, 42(6), 861865. https://doi.org/10.1037/h0037562

Beck, A. T., \& Steer, R. A. (1993). Manual for the Beck Hopelessness Scale (2. ${ }^{\text {a }}$ ed.). Psychological Corporation.

Bentler, P. M. (1990). Comparative Fit Indexes in Structural Models. Psychological Bulletin, 107(2), 238-246. https:// doi.org/10.1037/0033-2909.107.2.238
Bobes, J., Portilla, M., Bascarán, M., Sáiz, P., \& Bousoño, M. (2002). Banco de Instrumentos Básicos para la Práctica de la Psiquiatría Clínica. Ars Médica, Psiquiatría Editores.

Boduszek, D., \& Dhingra, K. (2016). Construct validity of the Beck Hopelessness Scale (BHS) among university students: A multitrait-multimethod approach. Psychological Assessment, 28(10), $1325 . \quad$ https://doi.org/10.1037/ pas0000245

Borges, G., Saltijeral, M. T., Bimbela, A., \& Mondragón, L. (2000). Suicide attempts in a simple of patients from a general hospital. Archives of medical Research, 31(4), 366372. https://doi.org/10.1016/S0188-4409(00)00079-5

Córdoba, O. M., \& Rosales, P. J. (2011). Consistencia interna y estructura factorial de la Escala de Desesperanza de Beck en estudiantes mexicanos. Revista de Psicología, 29(2), 289-309. http://www.scielo.org.pe/scielo. php?pid=S0254-92472011000200005\&script=sci_art text

Choi, J., Peters, M., \& Mueller, R. (2010). Correlational analysis of ordinal data: from Pearson's $r$ to Bayesian polychoric correlation. Asia Pacific Education Review, 11, 459-466. https://doi.org/10.1007/s12564-010-9096-y

Eremenco, S. L., Cella, D., \& Arnold, B. J. (2005). A comprehensive method for the translation and cross-cultural validation of health status questionnaires. Evaluation and the Health Professions, 28(1), 212-232. https://doi. org/10.1177/0163278705275342

Espinosa, J., Blum, B., \& Romero, P. (2009). Riesgo y letalidad suicida en pacientes con trastorno límite de la personalidad (TLP), en un hospital de psiquiatría. Salud Mental, 32(4), 317-325. https://www.medigraphic.com/pdfs/salmen/sam2009/sam094g.pdf

Fava, J. L., \& Velicer, W. F. (1992). The effects of overextraction on factor and component analysis. Multivariate Behavioral Research, 27(3), 387-415. https://doi.org/10.1207/ s15327906mbr2703_5

Freiberg, H. A., Stover, J. B., de la Iglesia, G., \& Fernández, L. M. (2013). Correlaciones policóricas y tetracóricas en estudios factoriales exploratorios y confirmatorios. Ciencias Psicológicas, VII(2), 151-164. http://www.scielo.edu.uy/ $\mathrm{pdf} / \mathrm{cp} / \mathrm{v} 7 \mathrm{n} 2 / \mathrm{v} 7 \mathrm{n} 2 \mathrm{a} 05 . \mathrm{pdf}$

Garrido, L. E., Abad, J., \& Ponsoda, V. (2011). Performance of Velicer's Minimum Average Partial factor retention method with categorical variables. Educational and Psychological Measurement, 71(3), 551-570. https://doi. org/10.1177/0013164410389489

Gheihman, G., Zimmermann, C., Deckert, A., Fitzgerald, P., Mischitelle, A., ... Rodin, G. (2016). Depression and hopelessness in patients with acute leukemia: the psychological impact of an acute and life-threatening disorder. Psycho-Oncology, 25(8), 979-989. https://doi.org/10.1002/ pon.3940

González, C.C. (2009). Propiedades psicométricas de la escala de desesperanza de Beck en una muestra bogotana. Psychología. 
Avances de la disciplina, 3(2), 17-30. https://biblat.unam. $\mathrm{mx} /$ hevila/PsychologiaAvancesdeladisciplina/2009/vol3/ no2/1.pdf

González, S., Pineda, A., \& Gaxiola, J. C. (2018). Depresión adolescente: factores de riesgo y apoyo social como factor protector. Universitas Psychologica, 17(3), 1-11. https:// doi.org/10.11144/Javeriana.upsy17-3.dafr

Hanna, D., White, R., Lyons, K., McParland, M. J., Shannon, C., \& Mulholland, C. (2011). The structure of the Beck Hopelessness Scale: a confirmatory factor analysis in UK students. Personality and Individual Differences, 51(1), 1722. https://doi.org/10.1016/j.paid.2011.03.001

Horwitz, A. G., Berona, J., Czyz, E. K., Yeguez, C. E., \& King, C. A. (2016). Positive and Negative Expectations of Hopelessness as Longitudinal Predictors of Depression, Suicidal Ideation, and Suicidal Behavior in High-Risk Adolescents. Suicide Life Treat Behavior, 47(2), 168-176. https://doi.org/10.1111/ sltb.12273

Hu, L., \& Bentler, P. M. (1999). Cutoff criteria for fit indexes in covariance structure analysis: Conventional criteria versus new alternatives. Structural Equations Modeling, 6(1), 1-55. https://doi.org/10.1080/10705519909540118

Ibarra, A. C., Peñaloza, I., Jiménez, G. A., \& Díaz, C. M. (2000). Desesperanza y depresión en sujetos hospitalizados por intento suicida. Psiquis (México), 9(4), 79-83.

Innamorati, M., Lester, D., Balsamo, M., Erbuto, D., Ricci, F., ... Pomipili, M. (2014). Factor Validity of the Beck Hopelessness Scale in Italian Medical Patients. Journal of Psychopathology Behavioral Assessment, 36, 300-307. https://doi.org/10.1007/s10862-013-9380-3

Klonsky, E. D., Kotov, R., Bakst, S., Rabinowitz, J., \& Bromet, E. J. (2012). Hopelessness as a predictor of attempted suicide among first admission patients with psychosis: a 10-year cohort study. Suicide \& Life-Threatening Behavior, 42(1), 1-10. https://doi.org/10.1111/j.1943-278X.2011.00066.x

Kocalevent, R. D., Finck, C., Pérez-Trujillo, M., Sautier, L., Zill, J., \& Hinz, A. (2017). Standardization of the Beck Hopelessness Scale in the general population. Journal of mental health, 26(6), 516-522. https://doi.org/10.1080/096 38237.2016.1244717

Lazarevich, I., Delgadillo, G. H., \& Rodríguez, J. H. (2009). Indicadores psicosociales de riesgo suicida en los estudiantes universitarios. Psiquis (México), 18(3), 71-79.

Madeira, N., Albuquerque, E., Santos, T., Mendes, A., \& Roque, M. (2011). Death ideation in cancer patients: contributing factors. Journal of Psychosocial Oncology, 29(6), 636-642. https://doi.org/10.1080/07347332.2011.615381

Meisenberg, G., \& Williams, A. (2008). Are acquiescent and extreme response styles related to low intelligence and education? Personality and Individual Differences, 44(7), 1539-1550. https://doi.org/10.1016/j.paid.2008.01.010

Mikulic, M., Cassullo, G., Crespi, M., \& Marconi, A. (2009). Escala de Desesperanza BHS (A. Beck, 1974): Estudio de las Propiedades Psicométricas y Baremización de la Adaptación Argentina. Anuario de Investigaciones de la Facultad de Psicología de la UBA, XVI, 365-373. https:// www.redalyc.org/pdf/3691/369139945034.pdf

Mitchell, A., Pössel, P., van Voorhees, B., \& Eaton, W. (2016). Associations of depression status and hopelessness with breast cancer: A 24-year follow-up study. Journal of Health Psychology, 22(10), 1322-1331. https://doi. org/10.1177/1359105315626998

Mondragón, L., Saltijera, M., Bimbela, A., \& Borges, G. (1998). La ideación suicida y su relación con la desesperanza, el abuso de drogas y alcohol. Salud Mental, 21(5), 20-27. http://www.revistasaludmental.com.mx/index.php/ salud_mental/article/view/718/717

Morales-Vives, F., \& Dueñas, J. M. (2018). Predicting suicidal ideation in adolescent boys and girls: the role of psychological maturity, personality traits, depression and life satisfaction. The Spanish Journal of Psychology, 21, E10. https://doi.org/10.1017/sjp.2018.12

Muthén, L. K., \& Muthén, B. O. (2006). Mplus user's guide (Versión 4). Muthén \& Muthén.

Muthén, L. K., \& Muthén, B. O. (2012). Mplus user's guide (Versión 7). Muthén \& Muthén.

Mystakidou, K., Parpa, E., Tsilika, E., Pathiaki, M., Hatzipli, I., Galanos, A., \& Vlahos, L. (2008). The experience of hopelessness in a population of Greek cancer patients receiving palliative care. International Journal of Social Psychiatry, 54(3), 262-271. https://doi.org/10.1177/0020764008089857

Nissim, R., Flora, D. B., Cribbie, R. A., Zimmermann, C., Gagliese, L., \& Rodin, G. (2010). Factor structure of the beck Hopelessness Scale in individuals with advanced cancer. Psycho-Oncology, 19(3), 255-263. https://doi. org/10.1002/pon. 1540

Pompili, M., Tatarelli, R., Rogers, J. R., \& Lester, D. (2007). The Hopelessness Scale: A factor analysis. Psychological Reports, $\quad 100(2), \quad 375-378 . \quad$ https://doi.org/10.2466/ pro.100.2.375-378

Quintanilla, R., Haro, L. P., Flores, M. E., Celis de la Rosa, A., \& Valencia, S. (2003). Desesperanza y tentativa suicida. Investigación en Salud, 5(2), 113-116. https://www.redalyc. org/articulo.oa?id=14250206

Ribeiro, J. D., Yen, S., Joiner, T., \& Siegler, I. C. (2015). Capability for suicide interacts with states of heightened arousal to predict death by suicide beyond the effects of depression and hopelessness. Journal of affective disorders, 188(1), 53-59. https://doi.org/10.1016/j.jad.2015.07.037

Satorres, E., Ros, L., Meléndez, J. C., Serrano, J. P., Latorre, J. M., \& Sales, A. (2018). Measuring elderly people's quality of life through the Beck Hopelessness Scale: a study with a Spanish sample. Aging \& mental health, 22(2), 239-244. https://doi.org/10.1080/13607863.2016.1247427

Savalei, V., \& Falk, C. (2014). Recovering substantive factor loadings in the presence of acquiescence bias: A 
comparison of three approaches. Multivariate Behavioral Research, 49(5), 407-424. https://doi.org/10.1080/002731 71.2014 .931800

Suárez-Colorado, Y., Palacio, J. Caballero-Domínguez, C., \& Pineda-Roa, C. (2019) Adaptación, validez de constructo y confiabilidad de la escala de riesgo suicida Plutchik en adolescentes colombianos. Revista Latinoamericana de Psicología, 51(3), 145-152. https://doi.org/10.14349/ rlp.2019.v51.n3.1

Steer, R. A., Beck, A, T., \& Brown, G. K. (1997). Factors of the Beck Hopelessness Scale: facts and artifacts? Multivariate Experimental Clinical Research, 11(3), 131-141. https:// psycnet.apa.org/record/1999-10676-003

Steer, R. A., Kumar, G., \& Beck, A, T. (1993). Self-reported suicidal ideation in adolescent psychiatric inpatients. Journal of Consulting and Clinical Psychology, 61(6), 1096-1099. https://doi.org/10.1037/0022-006X.61.6.1096

Tovar, J. G., Favela, A. G., \& Sánchez, R. I. (2019). Estructura interna de la Escala de Desesperanza para Adultos Mayores de México. Pensamiento psicológico, 17(1), 61-72. https:// doi.org/10.11144/Javerianacali.PPSI17-1.eied

Troister, T., D'Agata, M. T., \& Holden, R. R. (2015). Suicide risk screening: Comparing the Beck Depression InventoryII, Beck Hopelessness Scale, and Psychache Scale in undergraduates. Psychological assessment, 27(4), 1500. https:// doi.org/10.1037/pas0000126
Van de Vijver, F. J., \& Tanzer, N. K. (2004). Bias and equivalence in cross-cultural assessment: an overview. European Review of Applied Psychology, 54(2), 119-135. https://doi. org/10.1016/j.erap.2003.12.004

Vautier, S., \& Pohl, S. (2009). Do balanced scales assess bipolar constructs? The case of the STAI scales. Psychological Assessment, 21(2), 187-193. https://doi.org/10.1037/ a0015312

Vautier, S., Callahan, S., Moncany, D., \& Sztulman, H. (2004). A bi-stable view of single constructs measured using balanced questionaires: application to trait anxiety. Structural Equation Modeling: A Multidisciplinary Journal, 11(2), 261-271. https://doi.org/10.1207/s15328007sem1102_7

Wang, Y. Y., Jiang, N. Z., Cheung, E. F., Sun, H. W., \& Chan, R. C. (2015). Role of depression severity and impulsivity in the relationship between hopelessness and suicidal ideation in patients with major depressive disorder. Journal of affective disorders, 183, 83-89. https://doi.org/10.1016/j. jad.2015.05.001

Waszczuk, M. A., Coulson, A. E., Gregory, A., \& Eley, T. C. (2016). A longitudinal twin and sibling study of the hopelessness theory of depression in adolescence and young adulthood. Psychological medicine, 46(9), 1935-1949. https://doi.org/10.1017/S0033291716000489 